\title{
Cytokines and depression in cancer patients and caregivers
}

This article was published in the following Dove Press journal:

Neuropsychiatric Disease and Treatment

\author{
Madeline $\mathrm{Li}^{1,2}$ \\ Ekaterina Kouzmina ${ }^{3}$ \\ Megan McCusker' \\ Danielle Rodin ${ }^{4}$ \\ Paul C Boutros $3,5,6$ \\ Christopher J Paige ${ }^{6-8}$ \\ Gary Rodin ${ }^{1,2}$
}

'Princess Margaret Cancer Centre, Department of Supportive Care, University Health Network, Toronto, Ontario, Canada; ${ }^{2}$ Department of Psychiatry, University of Toronto, Toronto, Ontario, Canada; ${ }^{3}$ Informatics \& Biocomputing Program, Ontario Institute for Cancer Research, Toronto, Ontario, Canada; ${ }^{4}$ Department of Radiation Oncology, University of Toronto, Toronto, Ontario, Canada; ${ }^{5}$ Department of Pharmacology \& Toxicology, Toronto, Ontario, Canada; ${ }^{6}$ Department of Medical Biophysics, University of Toronto, Toronto, Ontario, Canada; ${ }^{7}$ Department of Immunology, University of Toronto, Toronto, Ontario, Canada; ${ }^{8}$ Princess Margaret Cancer Centre, University Health Network, Toronto, Ontario, Canada
Correspondence: Madeline Li Department of Supportive Care, 16-749 Princess Margaret Cancer Centre, 610 University Avenue, Toronto, ON M5G 2M9, Canada Tel + I 416946450 I ext 7505 Fax + I 4169462047 Email madeline.li@uhn.ca
Objective: A better understanding of the biobehavioral mechanisms underlying depression in cancer is required to translate biomarker findings into clinical interventions. We tested for associations between cytokines and the somatic and psychological symptoms of depression in cancer patients and their healthy caregivers.

Patients and methods: The GRID Hamilton Rating Scale for Depression (Ham-D) was administered to 61 cancer patients of mixed type and stage, 26 primary caregivers and 38 healthy controls. Concurrently, blood was drawn for multiplexed plasma assays of 15 cytokines. Multiple linear regression, adjusted for biobehavioral variables, identified cytokine associations with the psychological (Ham-Dep) and somatic (Ham-Som) subfactors of the Ham-D.

Results: The Ham-Dep scores of cancer patients were similar to their caregivers, but their Ham-Som scores were significantly higher (twofold, $p=0.016$ ). Ham-Som was positively associated with IL-1ra (coefficient: $1.27, p \leq 0.001$ ) in cancer patients, and negatively associated with IL-2 (coefficient: $-0.68, p=0.018$ ) in caregivers. Ham-Dep was negatively associated with IL-4 (coefficient: $-0.67, p=0.004$ ) in cancer patients and negatively associated with IL-17 (coefficient: $-1.81, p=0.002$ ) in caregivers.

Conclusion: The differential severity of somatic symptoms of depression in cancer patients and caregivers and the unique cytokine associations identified with each group suggests the potential for targeted interventions based on phenomenology and biology. The clinical implication is that depressive symptoms in cancer patients can arise from biological stressors, which is an important message to help destigmatize the development of depression in cancer patients.

Keywords: depression, psychological symptoms, somatic symptoms, cytokines, cancer, caregiver

\section{Introduction}

Despite decades of research, currently available antidepressants which are based on monoamine depletion theories achieve remission of depression in fewer than $40 \%$ of patients, ${ }^{1}$ in part due to an inadequate understanding of the pathophysiology of depression. Depression in the context of medical illnesses, such as cancer, has an even more limited evidence base for the effectiveness of existing antidepressants, with meta-analysis of the only five placebo-controlled randomized trials indicating no significant difference between groups. ${ }^{2}$

The inflammatory hypothesis of depression has emerged as a predominant explanatory theory for its underlying pathophysiology, ${ }^{3}$ although the precise pathways and biobehavioral mechanisms associated with it have not yet been elucidated. ${ }^{4}$ This hypothesis invokes a stress-activated production of pro-inflammatory cytokines, leading to alterations in tryptophan and monoamine metabolism, in autoregulatory hypothalamic-pituitary-adrenal (HPA) axis pathways, in neuronal excitotoxicity, 
and/or in brain trophic factors, all potentially contributing to the development of major depression. ${ }^{5}$ Such stress-activated inflammatory responses are likely to be triggered by cancer and its treatment.

Biological stressors, such as tumor cell burden, infection, or treatment-induced tissue destruction, are a clear source of inflammatory activation in cancer patients that may contribute to depression and other so-called sickness behaviors. ${ }^{6}$ Psychological stress may also precipitate inflammation, activating the HPA axis, increasing peripheral sympathetic tone, and stimulating pro-inflammatory cytokine release from immune cells..$^{5}$ The role of psychological stress in the inflammation-depression relationship has been recently examined in cancer patients, ${ }^{7}$ but it is not known whether physical and psychosocial stressors elaborate distinct inflammatory profiles. However, the heterogeneity of both cancer and depression complicates research in this field and may contribute to the variability in associations found between cytokines and depressive symptoms in cancer patients. ${ }^{8}$

A better understanding of the phenomenology of depressive symptoms in cancer patients is required to translate biomarker findings into clinical interventions. This includes distinguishing the inflammatory correlates of biological and psychological stress, and the somatic and psychological symptom domains of depression. This approach aligns with the Mental Health Research Domain Criteria (RDoC) project of the National Institute of Mental Health to classify mental disorders based on behavioral and neurobiological dimensions, rather than solely on descriptive phenomenology. ${ }^{9}$ This move to considering dimensions of depression, rather than categorical diagnoses, parallels the trend in symptom cluster research in cancer and may be of value in identifying specific pathophysiological pathways to depression. ${ }^{6}$

Comparing cytokine correlates of the somatic and psychological symptoms of depression in cancer patients, as well as in their healthy primary caregivers, may help to clarify their specificity. In noncancer patients, pro-inflammatory cytokines have been shown to have higher associations with somatic symptoms than psychological symptoms of depression. ${ }^{10-12}$ Caregivers of cancer patients are an informative comparison group, because they are subject to psychological stress related to illness in their spouses, but do not directly suffer from the biological stresses of cancer. Psychological stressors common to cancer patients and their caregivers include alterations in family role functioning and intimate relationships, fears related to changes in bodily appearance and physical suffering, alterations in anticipated life trajectory, and existential concerns. Caregivers must also contend with the multiple roles of caregiving, managing the demands of the family and household and often maintaining their own employment. Cancer caregivers manifest equivalent or higher levels of depressive symptoms compared with cancer patients ${ }^{13}$ and have been understudied in terms of inflammatory markers. However, most such investigations have been conducted in caregivers of dementia patients, whereas cancer caregivers tend to be a younger and healthier population. ${ }^{14}$

We have separately reported on cytokine associations with interview-diagnosed major depression in cancer patients. ${ }^{15}$ In this report, we describe cytokine associations with somatic and psychological symptoms of depression in these cancer patients and their healthy primary caregivers. We examined a broad suite of cytokines to identify biomarkers potentially contributing to depressive symptoms. Cancer patients were expected to present with higher somatic symptoms than caregivers, and general predictions were that different cytokines would be associated with depressive symptoms in both groups. Identification of distinct cytokine associations in these groups will help to elucidate biobehavioral mechanisms that may underlie the inflammatory hypothesis of depression and may reveal pathways that can be targeted in the treatment of the somatic and psychological symptoms of depression.

\section{Patients and methods Subjects}

Cancer patients and their healthy primary caregivers were recruited from the outpatient oncology and psychosocial oncology clinics at the Princess Margaret Cancer Centre, Toronto, Canada. Healthy individuals recruited through advertisements in the surrounding university and hospital networks were included as a comparison group. All study subjects provided informed consent for participation, and this study received research ethics board approval from the University Health Network, Toronto, Canada.

Exclusion criteria intended to minimize variability in cytokine levels and symptom presentation included brain malignancy or history of neurological illness or trauma, substance abuse or dependence within 1 year, immunization within 30 days, blood donation within 60 days, pregnancy or use of hormonal contraceptives within 3 months, and the presence of any psychiatric comorbidity (except depression) identified by the Mini International Neuropsychiatric Interview. ${ }^{16}$

A total of 61 cancer patients completed the study, where $31 \%$ (19/61) of whom were diagnosed with major depression using the Structured Clinical Interview for Diagnostic and Statistical Manual of Mental Disorders (DSM)-IV (SCID). ${ }^{17}$ 
Cancers were of mixed type and stage, with the majority recruited from either the gastrointestinal (38\%) or lung cancer clinics (19\%) and having late-stage disease (64\%). A total of 26 primary caregivers were recruited, of whom three were diagnosed with major depression. In addition, 38 healthy controls recruited through hospital notices completed the study.

\section{Assessments}

As a continuous measure of depression severity, all subjects were administered the 17-item GRID Hamilton Rating Scale for Depression (Ham-D) by a single trained research assistant, ${ }^{18,19}$ just prior to blood collection. The GRID Ham-D is a semi-structured interview version of the Ham-D, ${ }^{19}$ in which reliability has been improved by a symptom frequency by intensity scoring grid. A Ham-D cutoff score of 7 is used to screen for depression in psychiatric populations. ${ }^{20}$ The Ham-D has been found to have a four-factor structure comprising anxiety, depression, insomnia, and somatic domains. ${ }^{21}$ Only the depression (Ham-Dep: depressed mood, suicidal thoughts, guilt, work and interests, psychomotor retardation) and somatic (Ham-Som: gastrointestinal, general somatic, weight loss, sexual symptoms) subfactors were used in this study to represent psychological and somatic symptoms of depression, respectively.

Health behaviors, medical history (including illnesses recorded in the Charlson Comorbidity Index $[\mathrm{CCI}]^{22}$ ), demographic, and biobehavioral variables were collected on a study questionnaire, including frequency of smoking, alcohol use, caffeine consumption, and exercise habits. Medical variables for cancer patients were extracted from the electronic medical record, including body mass index (BMI), cancer type, stage, and treatment.

\section{Plasma cytokine measurement}

The following biobehavioral conditions (at the time of blood draw) were stipulated to decrease cytokine variability: ${ }^{23}$ 1) Females were required to be in the follicular phase of the menstrual cycle (confirmed by estradiol and progesterone levels on the day of testing); 2) no strenuous physical exercise and no alcohol consumption within the past 48 hours; 3 ) no acute or infectious illness, allergic reactions, physical injuries, or dental work within the past 2 weeks; 4) all blood samples were drawn between 8 am and $10 \mathrm{am}$, following a 12-hour overnight fast; and 5) blood samples were collected in EDTA Vacutainer ${ }^{\circledR}$ tubes (BD, Franklin Lakes, NJ, USA), with plasma separated within 2 hours of collection and frozen in aliquots at $-70^{\circ} \mathrm{C}$ until assay.
Multiplexed electrochemiluminescence cytokine immunoassays were performed using the Meso Scale Discovery (MSD) system and read on a Sector ${ }^{\circledR}$ Imager 2400 (Meso Scale Diagnostics, Rockville, MD, USA). All assays were conducted in duplicate wells with mean values analyzed, from a single manufacturer lot number, using singly thawed aliquots. A combination of the MSD standard T helper (Th)1/Th2 10-plex panel comprising IFN- $\gamma$, IL-1 $\beta$, IL-2, IL-4, IL-5, IL-8, IL-10, IL-12p70, IL-13, and TNF- $\alpha$ and customized cytokine kits comprising IL-6, IL-1ra, IL-12p40, IL-17, and IL-2R $\alpha$ were used. Samples measuring below the lower limit of quantification (LLOQ) for the assay were treated as 0 . The Th1/Th2 panel was selected to ensure a broad coverage of pro- and anti-inflammatory cytokines, and the additional cytokines for the customized kits were selected to investigate important recent pathways of interest (IL-17) ${ }^{24}$ or cytokines previously reported in the literature in association with depression (IL-6, IL-1ra, and IL-2R $\alpha$ ). ${ }^{25,26}$

\section{Statistical analysis}

The total sample size of 125 subjects (including cancer patients, caregivers, and healthy controls) was used a priori to establish a data analysis plan. The number of allowable statistical comparisons was predetermined based on the total sample size, with false discovery rate (FDR) correction used where specified.

All data were loaded into the $\mathrm{R}$ statistical environment (v.3.1.1). The descriptive characteristics of the sample were compared between cancer patients, caregivers, and healthy controls. Categorical variables were compared using the Pearson's chi-square test, while continuous variables were compared with one-way analysis of variance (ANOVA).

\section{Cytokine level analysis and visualization}

The cytokine concentration data were transformed into $\log _{2}$-space, and infinite values were removed by imposing a ceiling of 14 as the maximum of all finite cytokine levels. Five of 15 cytokines were selected for analysis in pro- to anti-inflammatory cytokine ratios: IL-6:IL-4, TNF- $\alpha$ :IL-4, IFN- $\gamma:$ IL-4, IL-6:IL-10, and IFN- $\gamma:$ IL-10. Levels of the 15 cytokines and five ratios were visualized in the $\mathrm{R}$ statistical environment using the lattice (v0.20-29) and latticeExtra (v0.6-26) R package. To determine whether there were significant differences between these levels in cancer patients vs healthy subjects, and caregivers vs healthy subjects, the two-sample non-paired $t$-test with Welch's correction for heteroscedasticity at the 0.95 confidence level was used, with FDR correction applied to the resulting $p$-values. 
The magnitude of the differences was evaluated by finding the fold difference of the cancer patients' and caregivers' mean cytokine levels relative to the healthy controls. Since age and sex may contribute to the differing cytokine levels in these groups, ${ }^{23}$ multiple logistic regression analysis was performed to adjust for these variables in group-wise comparisons, with FDR correction applied to the resulting $p$-values.

Cytokine levels and symptom domains of depression Ham-Dep and Ham-Som were used to study the relationships between cytokine levels and depressive symptom domains in cancer patients and caregivers. Multiple linear regression was performed to determine the coefficients, standard errors, and $p$-values that related each cytokine level or ratio to the Ham-D subfactor. This analysis was performed with the Ham-D subfactors as continuous variables. The cancer patient results were adjusted for the effects of age, sex, BMI, active treatment (dichotomized as "on" vs "off"), and cancer stage and type (treated as unordered factors). The caregiver results were adjusted for the effects of age and sex. The adjusted coefficients were visualized using dotmaps for cancer patients vs caregivers using lattice (v0.20-29) and latticeExtra (v0.6-26) packages in the R statistical environment (v.3.1.1).

\section{Results}

Demographic and biobehavioral variables across the samples are summarized in Table 1. Aside from medical comorbidity and exercise habits, there were no significant differences between the three groups.

\section{Depression subfactors in cancer patients and caregivers}

GRID Ham-D depression severity scores for cancer patients, caregivers, and healthy controls are summarized in Table 2. Mean total GRID Ham-D scores in both the cancer patient group (10.1 \pm 7.4$)$ and the caregiver group (7.3 \pm 7.4$)$ were above the typical screening cutoff score of $7,{ }^{21}$ and both were higher than that in the healthy control group (1.7 \pm 2.0$)$. Mean total GRID Ham-D scores between cancer patients and caregivers were not significantly different from each other $(p=0.11)$.

Mean psychological symptom scores (Ham-Dep scores) were not significantly different between cancer patients and caregivers (3.2 vs $2.7, p=0.57$ ), but the mean somatic symptom score (Ham-Som score) was higher in the cancer group (2.3 vs $1.2, p=0.016$ ). Among cancer patients, there were higher levels in both Ham-Som and Ham-Dep subfactor
Table I Characteristics of the sample population

\begin{tabular}{|c|c|c|c|c|}
\hline Characteristics & $\begin{array}{l}\text { Cancer } \\
\text { patients } \\
(n=6 I)\end{array}$ & $\begin{array}{l}\text { Caregivers } \\
(n=26)\end{array}$ & $\begin{array}{l}\text { Healthy } \\
\text { controls } \\
(n=38)\end{array}$ & $p$-value \\
\hline \multicolumn{5}{|l|}{ Demographics } \\
\hline Sex, female (\%) & $35(57)$ & $17(65)$ & $21(55)$ & 0.70 \\
\hline $\begin{array}{l}\text { Mean income, } \\
\text { CAD (SD) }\end{array}$ & $\$ 88.5 \mathrm{~K}(\$ 42 \mathrm{~K})$ & $\$ 99.8 \mathrm{~K}(\$ 48 \mathrm{~K})$ & $\mathrm{N} / \mathrm{A}$ & 0.29 \\
\hline $\begin{array}{l}\text { Mean age, years } \\
\text { (SD) }\end{array}$ & $55.5(11.6)$ & $56.3(12.0)$ & $43.5(11.7)$ & 0.36 \\
\hline \multicolumn{5}{|l|}{ Ethnicity, n (\%) } \\
\hline Caucasian & $49(80)$ & $2 I(8 I)$ & $24(63)$ & 0.081 \\
\hline Asian & $5(8)$ & $5(19)$ & $9(24)$ & \\
\hline Other & $7(12)$ & 0 & $5(13)$ & \\
\hline \multicolumn{5}{|c|}{ Biobehavioral variables } \\
\hline Mean BMI (SD) & $25.2(5.8)$ & N/A & $N / A$ & \\
\hline $\mathrm{CCl}(\mathrm{SD}) *$ & $5.0(2.2)$ & $2.3(0.6)$ & $2.0(0.2)$ & $<0.001$ \\
\hline Smokers (\%) & $8(13.1)$ & $3(11.5)$ & $6(15.8)$ & 0.94 \\
\hline $\begin{array}{l}\text { Alcohol (SD) } \\
\text { (drinks/week) }\end{array}$ & $2.71(5.47)$ & $3.56(5.13)$ & $2.01(2.66)$ & 0.62 \\
\hline $\begin{array}{l}\text { Caffeine (SD) } \\
\text { (drinks/week) }\end{array}$ & $21.5(22.5)$ & $20.7(14.1)$ & $15.9(12.5)$ & 0.59 \\
\hline $\begin{array}{l}\text { Exercise habits } \\
\text { (SD)** }\end{array}$ & $1.77(0.75)$ & $1.96(0.8 I)$ & $2.4 I(0.84)$ & 0.009 \\
\hline
\end{tabular}

Notes: $* \mathrm{CCl}$ calculated without age. $* *$ Mean (SD) exercise habits rated as: I, sedentary; 2, mild regular; 3, occasional vigorous; 4 , regular vigorous.

Abbreviations: BMI, body mass index; $\mathrm{CCl}$, Charlson Comorbidity Index; N/A, not available.

scores (3.5 \pm 2.2 and 6.5 \pm 3.4 , respectively) in those with major depression. Among subjects with major depression, cancer patients scored highest on the Ham-Som subfactor score (3.5 \pm 2.2$)$, while caregivers scored highest on the Ham-Dep subfactor score $(10.0 \pm 2.0)$.

\section{Cytokine associations with somatic and psychological symptoms of depression}

Cytokine associations with GRID Ham-D subfactors in cancer patients and caregivers are shown in Figure 1. In cancer patients, positive associations with somatic symptoms (HamSom) were found for IL-2R $\alpha$ (coefficient: $0.89, p=0.009$ ) and IL-1 ra (coefficient: $1.27, p \leq 0.001$ ). Caregivers showed a negative association between Ham-Som and IL-2 (coefficient: $-0.68, p=0.02$ ) and positive associations with ratios TNF- $\alpha$ :IL-4 (coefficient: $0.40, p \leq 0.001$ ) and IL-6:IL-4 (coefficient: 0.27, $p<0.001$ ).

Psychological symptoms of depression in cancer patients (Ham-Dep) were negatively associated with the antiinflammatory cytokine IL-4 (coefficient: $-0.67, p=0.004$ ) and with the pro-inflammatory cytokine IL-12p70 (coefficient: $-0.41, p=0.025$ ). Two cytokine ratios including IL-4 were positively associated with Ham-Dep in cancer patients: IL-6:IL-4 (coefficient: 0.28, $p=0.036$ ) and TNF- $\alpha$ :IL-4 (coefficient: $0.46, p=0.007)$. Among caregivers, the only significant 
Table 2 Ham-D scores across the sample population

\begin{tabular}{|c|c|c|c|c|c|c|}
\hline \multirow{2}{*}{$\begin{array}{l}\text { Sample population } \\
\text { Cancer patients }(n=6 I)\end{array}$} & \multicolumn{2}{|c|}{ Mean Ham-D total (SD) } & \multicolumn{2}{|c|}{ Mean Ham-Dep (SD) } & \multicolumn{2}{|c|}{ Mean Ham-Som (SD) } \\
\hline & & I0.I (7.4) & & $3.2(3.5)$ & & $2.3(2.1)$ \\
\hline Patients with MD (n=19) & $22.7(7.1)$ & & $6.5(3.4)$ & & $3.5(2.2)$ & \\
\hline Patients without MD ( $n=42)$ & $7.2(5.4)$ & & $1.7(2.3)$ & & $1.7(1.8)$ & \\
\hline Caregivers $(n=26)$ & & $7.3(7.4)$ & & $2.7(3.4)$ & & $1.2(1.4)$ \\
\hline Caregivers with MD (n=3) & $16.6(5.5)$ & & $10(2.0)$ & & $2.7(1.5)$ & \\
\hline Caregivers without MD (n=23) & $5.3(4.9)$ & & $1.8(2.2)$ & & $1.0(1.3)$ & \\
\hline Cancer vs caregiver ( $p$-value) & & 0.11 & & 0.57 & & 0.016 \\
\hline Healthy controls $(n=38)$ & $1.7(2.0)$ & & $0.53(0.98)$ & & $0.05(0.23)$ & \\
\hline
\end{tabular}

Notes: MD determined by SCID interview. Ham-Dep, depression subfactor of Ham-D; Ham-Som, somatic subfactor of Ham-D.

Abbreviations: Ham-D, Hamilton Rating Scale for Depression; MD, major depression; SCID, Structured Clinical Interview for DSM-IV.

cytokine relationship found with Ham-Dep was a negative association with IL-17 (coefficient: $-1.81, p=0.002$ ).

\section{Impact of statistical adjustments}

Table 3 describes the impact of adjustments in the psychological symptom (Ham-Dep) analyses in cancer patients and caregivers, where the strongest and most statistically significant cytokine associations were identified. The association of IL-17 with Ham-Dep in caregivers was controlled for sex and age, with sex not demonstrating a significant effect (coefficient: $-0.16, p=0.89$ ), while age had a small but significant effect (coefficient: $-0.13, p<0.006$ ).
A

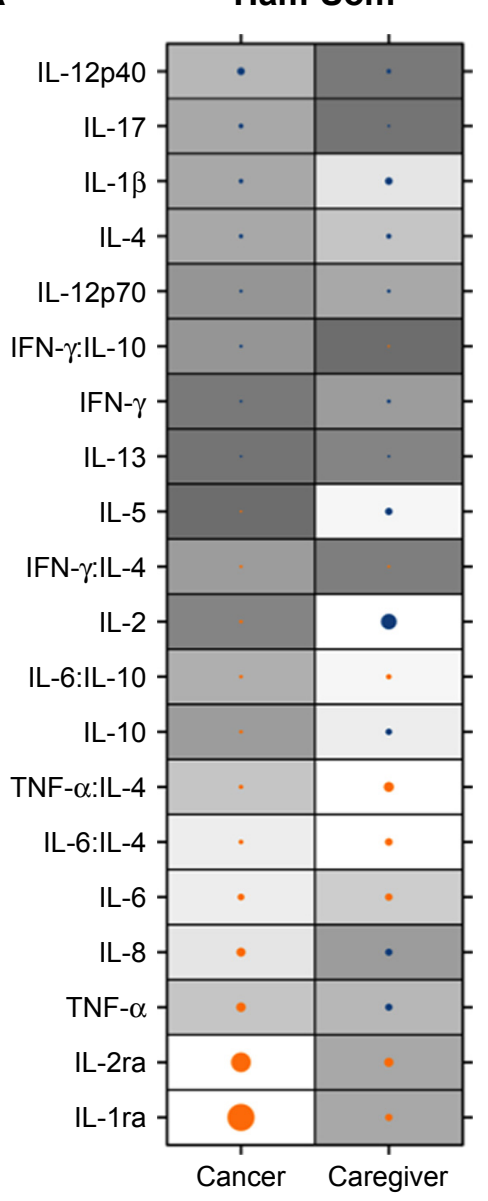

B

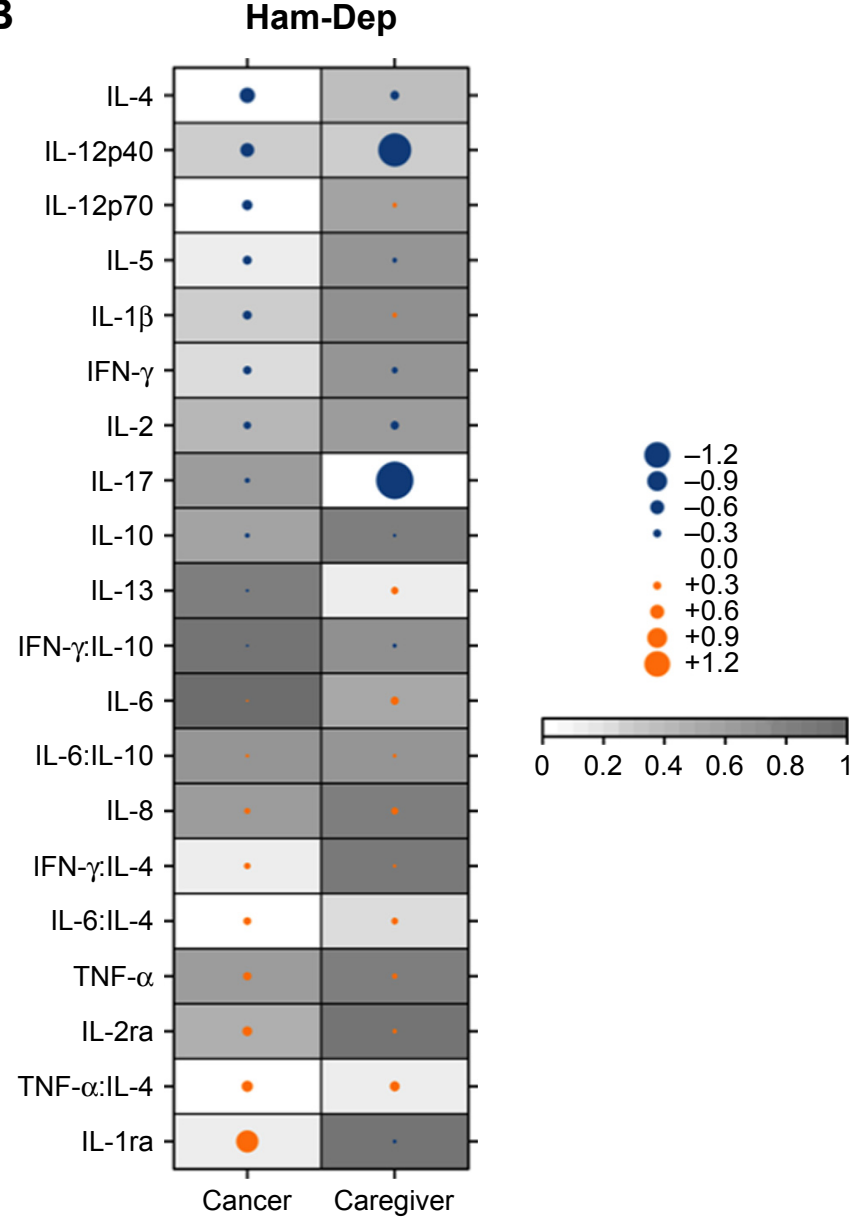

Figure I Cytokine associations in cancer patients and caregivers with depression subfactors.

Notes: (A) Dotmap of cytokine associations with Ham-Som scores. (B) Dotmap of cytokine associations with Ham-Dep scores. Coefficient strength is represented by the size of the circle (blue, negative: orange, positive), with the $p$-value represented in gray scale.

Abbreviations: Ham-D, Hamilton Rating Scale for Depression; Ham-Dep, depression subfactor of Ham-D; Ham-Som, somatic subfactor of Ham-D. 
Table 3 Impact of statistical adjustments on significant associations with Ham-Dep

\begin{tabular}{llll}
\hline Variable & Coefficient & $\begin{array}{l}\text { Standard } \\
\text { error }\end{array}$ & p-value \\
\hline IL-I7 in caregivers & & & \\
Age & -0.13 & 0.04 & 0.006 \\
Sex (female) & -0.16 & 1.06 & 0.89 \\
IL-I7 & $-\mathrm{I} .8 \mathrm{I}$ & 0.50 & 0.002 \\
IL-4 in cancer patients & & \\
Age & -0.08 & 0.04 & 0.034 \\
Sex (female) & -0.16 & 0.83 & 0.86 \\
BMI & 0.14 & 0.08 & 0.089 \\
Active treatment & 0.82 & 0.89 & 0.36 \\
Stage III/IV & -3.75 & 1.11 & 0.001 \\
Hematologic & -4.48 & 1.40 & 0.003 \\
IL-4 & -0.67 & 0.22 & 0.004 \\
\hline
\end{tabular}

Note: Ham-Dep, depression subfactor of Ham-D.

Abbreviations: BMI, body mass index; Ham-D, Hamilton Rating Scale for Depression.

For the IL-4 and Ham-Dep association in cancer patients, cancer stage and type had large and significant impacts (coefficient: $-3.75, p=0.001$ and coefficient: $-4.48, p=0.003$, respectively), but age, sex, BMI, or treatment did not.

\section{Cytokine levels in cancer patients, caregivers, and healthy controls}

The cytokine associations with depressive symptom scores did not always coincide with increased or decreased cytokine levels relative to healthy controls. As summarized in Table 4, the positive association with somatic symptoms coincided with higher plasma levels in cancer patients for IL-2R $\alpha$ (odds ratio [OR]: 4.01, $p=0.003$ ), but not for IL-1ra (OR: 1.30, $p=0.42$ ). Pro-inflammatory cytokines IL-2 (negatively associated with somatic symptoms in caregivers) and IL-12p70 (negatively associated with psychological symptoms in cancer patients) were increased in both caregivers (OR: 2.12, $p=0.009)$ and cancer patients (OR: $1.58, p=0.003)$, relative to healthy controls.

The strongest and most statistically significant associations identified were the negative associations with psychological symptoms for anti-inflammatory cytokine IL-4 in cancer patients and pro-inflammatory cytokine IL-17 in caregivers. IL-4 was significantly increased in cancer patients (OR: 1.93, $p \leq 0.001$ ), and IL-17 significantly decreased in caregivers (OR: $0.23, p=0.010$ ), relative to healthy controls (Table 4).

Positive associations of the IL-6:IL-4 and TNF- $\alpha$ :IL-4 ratios with somatic symptoms in caregivers and with psychological symptoms in cancer patients coincided with lower plasma levels of these ratios in both groups, compared with healthy controls; although in contrast to the single-cytokine between-group differences described earlier, the ORs were of lesser magnitude and of marginal statistical significance (ORs from 0.71 to $0.86, p$-values from 0.02 to 0.06 ; Table 4 ).

\section{Discussion}

In this study of cytokines and depressive symptoms in cancer patients and their caregivers, we found that cancer patients reported more somatic symptoms of depression. The higher somatic symptom scores in cancer patients with major depression could be explained by overlap with the symptoms of cancer and its treatment, but is also consistent with the known somatic symptom amplification of depression that has been shown to occur with depression. ${ }^{27}$ Both somatic and psychological symptoms were increased in cancer patients with major depression, compared to those without this psychological disturbance, providing support to retain both symptom types in the diagnosis of depression in cancer patients. ${ }^{28}$ However, the observed trend toward a differential severity of these symptom domains in cancer patients and

Table 4 Cytokine levels and ratios in cancer patients and caregivers compared with healthy controls

\begin{tabular}{|c|c|c|c|c|}
\hline \multirow[t]{2}{*}{ Cytokine } & \multicolumn{2}{|c|}{ Cancer patients vs healthy controls } & \multicolumn{2}{|c|}{ Caregivers vs healthy controls } \\
\hline & $\begin{array}{l}\text { Fold difference } \\
\text { ( } p \text {-value) }\end{array}$ & $\begin{array}{l}\text { OR } \\
\text { ( } p \text {-value) }\end{array}$ & $\begin{array}{l}\text { Fold difference } \\
\text { ( } p \text {-value) }\end{array}$ & $\begin{array}{l}\text { OR } \\
\text { ( } p \text {-value) }\end{array}$ \\
\hline IL-2 & $8.5 \mathrm{I}(<0.00 \mathrm{I})$ & $3.00(<0.00 \mathrm{I})$ & $4.14(<0.001)$ & $2.12(0.009)$ \\
\hline IL-17 & $1.60(0.002)$ & $1.57(0.091)$ & $-1.65(0.009)$ & $0.23(0.010)$ \\
\hline IL-4 & $9.25(<0.001)$ & $1.93(<0.001)$ & $8.22(<0.001)$ & $2.07(0.009)$ \\
\hline IL-I2p70 & $3.6 \mathrm{I}(<0.00 \mathrm{I})$ & $1.58(0.003)$ & $3.18(0.017)$ & $\mathrm{I} .45(0.028)$ \\
\hline IL-2R $\alpha$ & $\mathrm{I} .80(<0.00 \mathrm{I})$ & $4.01(0.003)$ & $-1.04(0.77)$ & $0.28(0.058)$ \\
\hline IL-I ra & $1.30(0.026)$ & $1.30(0.42)$ & $\mathrm{I} .09(0.6 \mathrm{I})$ & $0.7 \mathrm{I}(0.48)$ \\
\hline TNF- $\alpha: I L-4$ & $-4.06(<0.00 I)$ & $0.78(0.015)$ & $-3.97(0.006)$ & $0.71(0.042)$ \\
\hline IL-6:IL-4 & $-3.25(0.018)$ & $0.86(0.064)$ & $-6.15(0.007)$ & $0.77(0.038)$ \\
\hline
\end{tabular}

Notes: Fold differences between groups are given, with FDR-corrected $p$-values. Multiple regression ORs, adjusted for age and sex, are given, with FDR-corrected $p$-values. Abbreviations: FDR, false discovery rate; OR, odds ratio. 
caregivers suggests that different mechanistic pathways may underlie these symptoms in cancer patients and caregivers. A larger sample of depressed caregivers will be required to substantiate this possibility.

We identified a negative association between IL-4 and psychological symptoms of depression in cancer patients. Interestingly, a negative association with IL-4 was also the only significant cytokine association found for SCIDbased categorically defined major depression in this patient sample, ${ }^{15}$ suggesting that it may be the biological markers of the psychological symptoms that distinguish cancer patients with and without major depression. Evaluation of the impact of statistical control for biobehavioral variables on this association indicated that cancer stage and type overwhelm any effects of age, sex, BMI, or active treatment, which is an important consideration for future study designs.

In caregivers, psychological symptoms of depression were negatively associated with IL-17, and in this noncancer population, age was found to have a significant impact on the association. We speculate that the difference in cytokine associations with psychological symptoms of depression between cancer patients and caregivers may be related to cytokine network alterations related to cancer and cancer treatment, obscuring alterations evoked purely by psychological stress, as represented in the caregiver population.

IL-17 is the primary cytokine produced by Th17 cells, a highly pro-inflammatory effector $\mathrm{T}$ cell type that functions in autoimmunity. ${ }^{29}$ Th17 actions are countered by inflammationsuppressing regulatory T cells (Treg), with IL-6 promoting differentiation toward Th17. ${ }^{30}$ Thus, high levels of IL-6 may be a necessary but insufficient condition in the pathway to depression, playing more of a mediating role in the pathophysiology.

IL-6 has previously been reported in association with depression in cancer, particularly the somatic symptom domain. ${ }^{31-33}$ Studies in noncancer populations have also reported positive associations of IL- 6 , as well as TNF- $\alpha$ and sIL-2R (soluble IL-2R $\alpha$ ) with somatic symptoms of major depression. ${ }^{10,11}$ Somatic symptoms have also been found to be predictive of increased TNF- $\alpha$ levels in women with major depression, but not men. ${ }^{12}$ Somatic symptoms in this study were positively associated with IL-2R $\alpha$ and IL-1 ra in cancer patients, and negatively associated with IL-2 in caregivers. The differences between our results and those of other studies may be due to differences in the clinical population, the careful attention to biobehavioral confounds which sometimes lacking in other studies, or differences in the construct validity of the somatic symptom measures used.
Cytokines are known to have pleiotropic functions and operate in interactive networks, reciprocally influencing the expression of each other. ${ }^{34}$ Their pro-inflammatory functions inherently trigger a compensatory anti-inflammatory reflex system (CIRS), ${ }^{35}$ with the balance between the two determining net inflammatory effects. Such network complexities may be the basis for our finding of a positive association between IL2-R $\alpha$, but a negative association with its IL-2 ligand and somatic symptoms in caregivers. Our exploratory analyses of inflammatory:anti-inflammatory cytokine ratios also revealed positive associations of both IL-6:IL-4 and TNF- $\alpha$ :IL-4 with psychological symptoms in cancer patients, but with somatic symptoms in caregivers. These findings support our hypothesis that the biobehavioral mechanisms and cytokine profiles associated with depression would be distinct between the two groups. What might account for the apparent divergence in symptom domains associated with these cytokines and ratios between cancer patients and caregivers is unclear, highlighting it as an area of interest for future study in a larger, networked analysis.

Anti-inflammatory approaches for the treatment of depression are now being explored. ${ }^{36}$ Studies suggest that nonsteroidal anti-inflammatory drugs (NSAIDs) given solely or as adjuncts to antidepressants are more effective than placebo in treating the symptoms of depression. ${ }^{37}$ Whereas NSAIDs are broad-acting agents, inhibitors targeting specific cytokine pathways such as TNF- $\alpha$ and IL- 6 have been shown to improve the symptoms of depression in patients with rheumatic and other inflammatory conditions. ${ }^{38}$ Some studies have also suggested that antidepressants potentially have anti-inflammatory properties, ${ }^{37}$ and antidepressant response has been associated with reductions in pro-inflammatory cytokines such as IL-1 $\beta$, TNF- $\alpha$, and IL- $6 .{ }^{39}$

However, antidepressant effects of anti-inflammatory agents must be explored with an understanding of the underlying biobehavioral mechanisms in individual patients. Raison et $\mathrm{a}^{40}$ reported an antidepressant effect of infliximab, a TNF- $\alpha$ antagonist, but only in the subgroup of patients with higher baseline inflammation as measured by high levels of peripheral C-reactive protein. Potential specific biobehavioral mechanisms identified in this study include a negative association between psychological symptoms of depression with IL-4 in cancer patients and IL-17 in caregivers, whereas somatic symptoms of depression were positively associated with IL-2R $\alpha$ and IL-1ra in cancer patients and negatively associated with IL-2 in caregivers.

Limitations of the current study include the correlational nature of the reported associations, which do not permit 
causal interpretations, and the low number of caregivers with major depression recruited. Recruitment of sufficient numbers of cancer patients with major depression is often a significant barrier in biomarker studies, and recruitment of depressed caregivers may be an even greater challenge. In addition, BMI data were extracted from the electronic medical record and were therefore not available to include in statistical adjustments for caregivers or healthy controls.

\section{Conclusion}

This study found distinct cytokine associations with HamD-defined somatic and psychological symptom domains of depression in cancer patients and caregivers, suggesting that there may be distinct therapeutic targets for these symptom domains in these populations. This also suggests that there are different pathways to depressive symptoms in cancer patients and caregivers, which may be differentially precipitated by biological and psychological stressors. The clinical implication of such findings is that depressive symptoms in cancer patients can be precipitated by biological stressors, an important message to help de-stigmatize the emergence of depression in cancer patients.

\section{Disclosure}

The authors report no conflicts of interest in this work.

\section{References}

1. Mohamed S, Johnson GR, Chen P, et al. Effect of antidepressant switching vs augmentation on remission among patients with major depressive disorder unresponsive to antidepressant treatment: the VAST-D randomized clinical trial. JAMA. 2017;318(2):132-145.

2. Li M, Kennedy EB, Byrne N, et al. Systematic review and metaanalysis of collaborative care interventions for depression in patients with cancer. Psychooncology. 2017;26(5):573-587.

3. Li M, Soczynska JK, Kennedy SH. Inflammatory biomarkers in depression. An opportunity for novel therapeutic interventions. Curr Psychiatry Rep. 2011;13(5):316-320.

4. Low CA, Bovbjerg DH. Depressive symptoms in patients with cancer. Does cortisol keep cytokines from singing the blues? Psychosom Med. 2014;76(4):248-251.

5. Miller AH, Maletic V, Raison CL. Inflammation and its discontents: the role of cytokines in the pathophysiology of major depression. Biol Psychiatry. 2009;65(9):732-741.

6. Dantzer R, Meagher MW, Cleeland CS. Translational approaches to treatment-induced symptoms in cancer patients. Nat Rev Clin Oncol. 2012;9(7):414-426.

7. Wu SM, Yang HC, Thayer JF, Anderson BL. Association of the physiological stress response with depressive symptoms in patients with breast cancer. Psychosom Med. 2014;76(4):252-256.

8. Miller AH, Ancoli-Israel S, Bower JE, Capuron L, Irwin MR. Neuroendocrine-immune mechanisms of behavioral comorbidities in patients with cancer. J Clin Oncol. 2008;26(6):971-982.

9. Cuthbert BN. The RDoC framework: facilitating transition from ICD/ DSM to dimensional approaches that integrate neuroscience and psychopathology. World Psychiatry. 2014;13(1):28-35.
10. Euteneuer F, Schwarz MJ, Dannehl K, Hartung A, Westermann S, Rief W. Increased soluble interleukin-2 receptor levels are related to somatic but not to cognitive-affective features in major depression. Brain Behav Immun. 2012;26(8):1244-1248.

11. Duivis HE, Vogelzangs N, Kupper N, de Jonge P, Penninx BW. Differential association of somatic and cognitive symptoms of depression and anxiety with inflammation: findings from the Netherlands Study of Depression and Anxiety (NESDA). Psychoneuroendocrinology. 2013; 38(9):1573-1585.

12. Dannehl K, Rief W, Schwarz MJ, et al. The predictive value of somatic and cognitive depressive symptoms for cytokine changes in patients with major depression. Neuropsychiatr DisTreat. 2014;10:1191-1197.

13. Braun M, Mikulincer M, Rydall A, Walsh A, Rodin G. Hidden morbidity in cancer: spouse caregivers. J Clin Oncol. 2007;25(30):4829-4834.

14. Rohleder N, Marin TJ, Ma R, Miller GE. Biologic cost of caring for a cancer patient: dysregulation of pro- and anti-inflammatory signaling pathways. J Clin Oncol. 2009;27(18):2909-2915.

15. Li M, Kouzmina E, McCusker M, et al. Pro- and anti-inflammatory cytokine associations with major depression in cancer patients. Psychooncology. Epub 2016 Nov 15:doi:10.1002/pon.4316.

16. Sheehan DV, Lecrubier Y, Sheehan KH, et al. The Mini-International Neuropsychiatric Interview (M.I.N.I.): the development and validation of a structured diagnostic psychiatric interview for DSM-IV and ICD-10. J Clin Psychiatry. 1998;59(suppl 20):22-33.

17. First MB, Spitzer RL, Gibbon M, Williams JBW. Structured Clinical Interview for DSM-IV Axis I Disorders. Clinician Version (SCID-CV). Washington, DC: American Psychiatric Press. Inc.; 1996.

18. Tabuse H, Kalali A, Azuma H, et al. The new GRID Hamilton Rating Scale for Depression demonstrates excellent inter-rater reliability for inexperienced and experienced raters before and after training. Psychiatry Res. 2007;153(1):61-67.

19. Williams JB, Kobak KA, Bech P, et al. The GRID-HAMD: standardization of the Hamilton Depression Rating Scale. Int Clin Psychopharmacol. 2008;23(3):120-129.

20. Hamilton M. A rating scale for depression. JNeurol Neurosurg Psychiatry. 1960;23:56-62.

21. Shafer AB. Meta-analysis of the factor structures of four depression questionnaires: Beck, CES-D, Hamilton, and Zung. J Clin Psychol. 2006; 62(1):123-146.

22. Charlson ME, Pompei P, Ales KL, MacKenzie CR. A new method of classifying prognostic comorbidity in longitudinal studies: development and validation. J Chronic Dis. 1987;40(5):373-383.

23. O'Connor MF, Bower JE, Cho HJ, et al. To assess, to control, to exclude: effects of biobehavioral factors on circulating inflammatory markers. Brain Behav Immun. 2009;23(7):887-897.

24. Chen Y, Jiang T, Chen P, et al. Emerging tendency towards autoimmune process in major depressive patients: a novel insight from Th17 cells. Psychiatry Res. 2011;188(2):224-230.

25. Dowlati Y, Herrmann N, Swardfager W, et al. A meta-analysis of cytokines in major depression. Biol Psychiatry. 2010;67(5):446-457.

26. Liu Y, Ho RC, Mak A. Interleukin (IL)-6, tumour necrosis factor alpha (TNF- $\alpha$ ) and soluble interleukin-2 receptors (sIL-2R) are elevated in patients with major depressive disorder: a meta-analysis and metaregression. J Affect Disord. 2012;139(3):230-239.

27. Fitzgerald P, Lo C, Li M, Gagliese L, Zimmerman C, Rodin G. The relationship between depression and physical symptom burden in advanced cancer. BMJ Support Palliat Care. 2015;5(4):381-388.

28. Mitchell AJ, Lord K, Symonds P. Which symptoms are indicative of DSMIV depression in cancer settings? An analysis of the diagnostic significance of somatic and non-somatic symptoms. J Affect Disord. 2012; 138(1-2):137-148.

29. Mills KH. Induction, function and regulation of IL-17-producing T cells. Eur J Immunol. 2008;38(10):2636-2649.

30. Naugler WE, Karin M. The wolf in sheep's clothing: the role of interleukin-6 in immunity, inflammation and cancer. Trends Mol Med. 2008;14(3):109-119. 
31. Lutgendorf SK, Weinrib AZ, Penedo F, et al. Interleukin-6, cortisol, and depressive symptoms in ovarian cancer patients. J Clin Oncol. 2008; 26(29):4820-4827.

32. Inagaki M, Akechi T, Okuyama $\mathrm{T}$, et al. Associations of interleukin-6 with vegetative but not affective depressive symptoms in terminally ill cancer patients. Support Care Cancer. 2013;21(8):2097-2106.

33. Breitbart W, Rosenfeld B, Tobias K, et al. Depression, cytokines, and pancreatic cancer. Psychooncology. 2014;23(3):339-345.

34. Kopf M, Bachmann MF, Marsland BJ. Averting inflammation by targeting the cytokine environment. Nat Rev Drug Discov. 2010;9(9): 703-718.

35. Maes M, Berk M, Goeler L, et al. Depression and sickness behaviour are Janus-faced responses to shared inflammatory pathways. BMC Med. 2012;10:66.

36. Kopschina Feltes P, Doorduin J, Klein HC. Anti-inflammatory treatment for major depressive disorder: implications for patients with an elevated immune profile and non-responders to standard antidepressant therapy. J Psychopharmacol. 2017;31(9):1149-1165.
37. Schmidt FM, Kirkby KC, Lichtblau N. Inflammation and immune regulation as potential drug targets in antidepressant treatment. Cur Neurophamacol. 2016;14(7):674-687.

38. Kappelmann N, Lewis G, Dantzer R, Jones PB, Khandaker GM. Antidepressant activity of anti-cytokine treatment: a systematic review and meta-analysis of chronic inflammatory conditions. Mol Psychiatry. Epub 2016 Oct 18:1-9.

39. Hannestad J, DellaGioia N, Bloch M. The effect of antidepressant medication treatment on serum levels of inflammatory cytokines: a metaanalysis. Neuropsychopharmacology. 2011;36(12):2452-2459.

40. Raison CL, Rutherford RE, Woolwine BJ, et al. A randomized controlled trial of the tumor necrosis factor antagonist infliximab for treatmentresistant depression: the role of baseline inflammatory biomarkers. JAMA Psychiatry. 2013;70(1):31-41.
Neuropsychiatric Disease and Treatment

\section{Publish your work in this journal}

Neuropsychiatric Disease and Treatment is an international, peerreviewed journal of clinical therapeutics and pharmacology focusing on concise rapid reporting of clinical or pre-clinical studies on a range of neuropsychiatric and neurological disorders. This journal is indexed on PubMed Central, the 'PsycINFO' database and CAS,

\section{Dovepress}

and is the official journal of The International Neuropsychiatric Association (INA). The manuscript management system is completely online and includes a very quick and fair peer-review system, which is all easy to use. Visit http://www.dovepress.com/testimonials.php to read real quotes from published authors.

Submit your manuscript here: http://www.dovepress.com/neuropsychiatric-disease-and-treatment-journal 